\title{
$\infty$ \\ Riscos AsSOCIADOS AO USO INDISCRIMINADO DE VITAMINA D
}

\section{Autores}

Joao Filipe Costa Alves Pereira ${ }^{1}$, Priscilla Monteiro Quintino ${ }^{1}$, Renato Demarchi Foresto ${ }^{1}$, José MedinaPestana $^{1,2}$

\section{Afiliaçóes:}

${ }^{1}$ Fundação Oswaldo Ramos, Hospital do Rim, Departamento de Transplante, São Paulo, Brasil

${ }^{2}$ Universidade Federal de São Paulo, Departamento de Medicina, Disciplina de Nefrologia, São Paulo, Brasil

\section{Orcid}

Joao Filipe Costa Alves Pereira https://orcid.org/0000-0002-2871-174X

Priscilla Monteiro Quintino https://orcid.org/0000-0001-5916-5465

Renato Demarchi Foresto https://orcid.org/0000-0001-9370-0265 José Medina-Pestana https://orcid.org/0000-0002-0750-7360

\section{Autor Correspondente:}

Renato Demarchi Foresto

Fundação Oswaldo Ramos - Hospital do Rim Rua Borges Lagoa, 960 - Vila Clementino. E-mail: rdforesto@gmail.com

\section{RESUMO}

O uso de vitamina $\mathrm{D}$ como suplemento tem se popularizado por prescrição ou por automedicação e tem se tornado mais frequentes na literatura os casos de intoxicação. Descrevemos aqui 4 casos de pacientes que foram internados com intoxicação por vitamina $\mathrm{D}$ por diferentes formas de uso.

Palavras Chaves: colecalciferol, vitamina D, intoxicação por vitamina $\mathrm{D}$

\section{INTRODUÇÁO}

A vitamina $\mathrm{D}$ é consumida em duas principais formas: ergocalciferol (vitamina D2) e colecalciferol (vitamina D3). Ambas podem ser encontradas em suplementos vitamínicos e, em pequena quantidade, em alguns alimentos, como gema de ovo e peixes (1).

A principal fonte de vitamina $\mathrm{D}$ no organismo humano é endógena, através da metabolização de 7-dehidrocolesterol, na derme via exposição à luz solar (2). É importante lembrar que a exposição prolongada da pele à luz solar não produz quantidades tóxicas de vitamina $\mathrm{D} 3$, devido à conversão de parte da vitamina D3 sintetizada em metabólitos 
inativos. Além disso, a luz solar induz a produção de melanina, o que reduz a produçáo cutânea de vitamina D3 (3).

Quando obtida por fontes exógenas, a vitamina $\mathrm{D}$ consumida sofre hidroxilação no fígado, transformando-se na 25-hidroxivitamina D3. Esta, liga-se à proteína ligadora de vitamina $\mathrm{D}$ e é submetida, no tecido renal, à 1-alfa-hidroxilação para produzir a 1,25-hidroxivitamina D3 (4). A CYP24A1 [1,25(OH)2D24-hidroxilase] é a enzima responsável pela inativaçáo da 1,25-hidroxivitamina D3 em ácido calcitroico. Mutaçôes que levam à perda de função da CYP24A1 estáo associadas à hipercalcemia, hipercalciúria e nefrolitíase $(5,6)$.

Diversas condiçôes clínicas podem se beneficiar da correçâo de níveis muito baixos de vitamina $\mathrm{D}$, como idosos com osteoporose, mulheres gestantes, hiperparatireoidismo, sarcopenia, quedas recorrentes, hiperparatireoidismo secundário na doença renal crônica, o diabetes melito tipo 1, síndromes malabsortivas (7).

Apesar da associação entre níveis de vitamina $\mathrm{D}$ com eventos clínicos e da plausibilidade biológica, ainda é fraca a evidência de associação causal e de benefício consistente da correçấo da deficiência de vitamina D em diversas situaçôes (7).

É provável que o benefício da suplementação de vitamina $\mathrm{D}$ seja menor do que o inicialmente imaginado (8). Mas a dosagem de vitamina $\mathrm{D}$ é cada vez mais frequente (9) e alguns profissionais têm prescrito dosagens elevadas de vitamina $\mathrm{D}$ com relatos de intoxicação (10).

Ademais, vendida sem prescrição, seu uso tem se tornado mais comum ao longo dos anos (11) e relatos de intoxicação por uso de suplementos têm se tornado mais frequentes (12).

\section{Material e Métodos}

Foi realizada a descrição dos casos a partir da revisão de prontuário dos pacientes.

\section{Relato De CASOS}

\section{Caso 1}

Mulher de 45 anos, comerciante e procedente de São Paulo, refere ter utilizado, por orientação médica, duas ampolas de colecalciferol (50.000 UI/ ampola) associadas a quatro ampolas de testosterona intramuscular, no período de 1 ano. Posteriormente, iniciou reposição oral de colecalciferol (7000 UI/dia durante 30 dias) e reposição de cálcio (carbonato de cálcio $1 \mathrm{~g} / \mathrm{dia}$ ) nos três meses seguintes. Apresentava, como antecedentes pessoais, hipotireoidismo tratado com levotiroxina e nefrectomia parcial esquerda por nódulo. Negava osteoporose ou deficiência de vitamina $\mathrm{D}$. 
Referia história de perda de apetite, sede excessiva (ingestão de mais de 6 litros de água por dia), náusea, fraqueza, sonolência, episódios de perda de memória e obstipação intestinal iniciados há cerca de 3 semanas. Procurou a unidade de pronto-atendimento por dor abdominal de início súbito, à esquerda, do tipo pontada, além de piora dos sintomas anteriormente referidos. Ao exame físico, a paciente estava em regular estado geral e desidratada, sem outros achados.

A ultrassonografia de abdome não evidenciou alterações. Identificadas, entretanto, alterações da creatinina e hipercalcemia. Diante da história de consumo de doses elevadas de colecalciferol, foi solicitada a dosagem da 25-hidroxivitamina D (25OHD) que se encontrava elevada.

O diagnóstico de intoxicação por vitamina $\mathrm{D}$ foi confirmado e a paciente foi internada para tratamento com hidratação vigorosa e furosemida, devido à hipercalcemia grave. A reposição de vitamina $\mathrm{D}$, cálcio e testosterona foi suspensa no momento da admissão hospitalar.

A paciente evoluiu com melhora clínica e laboratorial, recebendo alta após 11 dias, assintomática e com melhora dos exames laboratoriais. Após 12 meses do diagnóstico, mantinha-se bem clinicamente, com exames laboratoriais mostrando recuperação da função renal, normocalcemia, PTH normal e níveis de $25 \mathrm{OHD}$ ainda elevados, porém com tendência à queda (tabela 1).

\section{Caso 2}

Homem, 78 anos, professor aposentado, relatou quadro de fraqueza iniciada há dois meses, associada à perda ponderal de cerca de $10 \mathrm{Kg}$. Possuía antecedentes de hiperplasia prostática benigna, hipotireoidismo, déficit auditivo bilateral, tromboembolismo pulmonar, fibrilação atrial e edema agudo de pulmão por insuficiência cardíaca. Em consulta de rotina, foi constatada alteração da função renal (creatinina $1,60 \mathrm{mg} / \mathrm{dL}$ ), atribuída, inicialmente, à associação de enalapril e espironolactona, porém, na ausência de melhora após suspensão das medicações, o paciente foi encaminhado para avaliação com nefrologista. Relatou que há 1 ano e 6 meses, fazia uso diário de 1 a 2 cápsulas de fórmula vitamínica composta por colecalciferol $(5.000$ UI/ cápsula). Ao exame físico, não se observaram outras anormalidades, além de sinais de desidratação. Foram realizados exames que identificaram alteração da creatinina $(4,2 \mathrm{mg} / \mathrm{dL})$ e hipercalcemia (cálcio iônico Cai: 1,99 mmol/L). Os exames laboratoriais iniciais estão descritos na tabela 2 .

Diante da suspeita de intoxicação por vitamina $\mathrm{D}$, foi solicitada a dosagem da 25OHD. Confirmado o diagnóstico, o paciente foi orientado a suspender o colecalciferol e prontamente submetido à hidratação e furosemida endovenosas. Recebeu uma dose de pamidronato $60 \mathrm{mg}$ durante a internação hospitalar. Foram descartadas outras 
causas de hipercalcemia, como neoplasia e mieloma múltiplo.

O paciente evoluiu com melhora clínica e progressiva melhora laboratorial. Recebeu alta após 10 dias, assintomático e com níveis de cálcio iônico, $25 \mathrm{OHD}$ e creatinina em queda. Após a alta, em retornos ambulatoriais, o paciente manteve-se bem clinicamente e exames laboratoriais mostraram normocalcemia e $25 \mathrm{OHD}$ dentro dos valores de referência.

\section{Caso 3}

Mulher, 86 anos, levada ao PS por quadro de hipoatividade e diarreia, sem febre ou outrossintomasassociados. Haviaapresentado queda da própria altura poucos dias antes $\mathrm{da}$ admissão. Familiares informaram que, antes deste quadro, a paciente não apresentava quaisquer alterações no nível de consciência. Referiam antecedente de hipertensão arterial sistêmica (HAS) bem controlada, com uso de nifedipino. À admissão hospitalar, foram realizados exames laboratoriais que identificaram: creatinina: $1,45 \mathrm{mg} / \mathrm{dL}$ (creatinina prévia: $0.8 \mathrm{mg} / \mathrm{dL}$ ), sódio: $134 \mathrm{mmol} / \mathrm{L}$, potássio: $2,8 \mathrm{mEq} / \mathrm{L}$ e Cai: $1,88 \mathrm{mmol} / \mathrm{L}$. Foi solicitada tomografia de crânio que se apresentava dentro dos parâmetros de normalidade para a faixa etária. Durante a investigação de hipercalcemia, identificou-se nível de 25OHD: > 160 pg/mL e PTH: 16,1 pg/mL. Instituído o tratamento para hipercalcemia com hidratação e pamidronato, a paciente evoluiu com melhora progressiva. Inicialmente os familiares negaram reposição de vitamina D. Entretanto, a paciente informou que no último mês havia utilizado suplemento vitamínico com alta dose de colecalciferol (50.000 UI/dia). Após compensação clínica, a paciente recebeu alta com orientaçóes de manter dieta pobre em cálcio e encaminhada para acompanhamento ambulatorial. Ainda durante a internação, apresentou melhora da função renal (creatinina retornou ao nível basal) e dos níveis séricos de cálcio.

Após 4 meses de acompanhamento, manteve-se assintomática, com Cai: $1,29 \mathrm{mmol} / \mathrm{L}$. Os valores de 25OHD permaneceram $>160$ $\mathrm{pg} / \mathrm{mL}$.

\section{Caso 4}

Homem, 30 anos, hígido e fisiculturista, referia uso de anabolizantes (esteroides sintéticos e GH) regularmente nos últimos três anos. Há 1 mês da admissão, realizou aplicação intramuscular de composto de vitaminas A, D e E. Procurou o PS por inapetência, náusea e poliúria. À admissão, apresentavase desidratado. Os exames laboratoriais identificaram: creatinina: $3.1 \mathrm{mg} / \mathrm{dL}$ e Cai: 2,03 $\mathrm{mmol} / \mathrm{L}$. Dosada a $25 \mathrm{OH}$ D: $>160 \mathrm{pg} / \mathrm{mL}$ e PTH: 9 pg/mL. Foram realizadas medidas para hipercalcemia e, como a creatinina permaneceu alterada mesmo após a correçáo do cálcio, optado por biópsia renal. Esta identificou infiltrado inflamatório compatí- 
vel com nefrite intersticial aguda. Iniciado o tratamento com corticoide, o paciente recebeu alta após melhora clínica e laboratorial. Após 18 meses de seguimento ambulatorial, apresentava função renal normal e $25 \mathrm{OHD}$ : $30 \mathrm{ng} / \mathrm{mL}$.

\section{Discussão}

A intoxicação por vitamina D é uma condição grave e potencialmente ameaçadora à vida, que ocorre quando a dosagem de vitamina $\mathrm{D}$ supera $150 \mathrm{ng} / \mathrm{mL}$ e cursa com hipercalcemia grave e alteração de função renal. Por ser lipofílica, a vitamina $D$ fica depositada em tecido adiposo e eventos como a intoxicação podem levar a níveis persistentemente elevados (3). Como pudemos observar nos casos descritos, ela pode ocorrer tanto pela administração de altas dosagens de reposição de vitamina $\mathrm{D}$ por prescrição uma estratégia cujos benefícios são altamente questionáveis e os potenciais malefícios bastante significativos, quanto por uso de reposição não supervisionada (9).

Com base em estudos preliminares e plausibilidade biológica, já se postulou que poderia haver benefício no uso de altas dosagens de vitamina $\mathrm{D}$ em algumas condiçóes clínicas. Mas esse benefício não se confirmou e o uso de altas doses de vitamina $\mathrm{D}$ pode ser deletério, podendo estar associado a um aumento no risco de quedas e fraturas (13) ou de formação de cálculos urinários em mulheres (14).

A recomendação de reposição de vitamina $\mathrm{D}$ deve ser, portanto, indicada de forma racional, monitorada e interrompida quando a deficiência da vitamina for corrigida. A SBEM recomenda que, pela falta de evidência de benefício e potencial de intoxicação, as concentraçôes não devem ser maiores que $60 \mathrm{ng} / \mathrm{mL}(7)$.

Outra forma de intoxicação por vitamina $\mathrm{D}$ é pela automedicação e consumo de suplementos vitamínicos por iniciativa própria (3). A automedicação é crescentemente um problema de saúde pública e carrega não somente os riscos de uso por um autodiagnóstico impreciso, mas também do uso de doses e tempo de tratamento diversos daqueles que seriam apropriados ao caso. Ademais, é frequente que drogas iniciadas por automedicação sejam "ocultadas" de profissionais de saúde (15). Isso é bem ilustrado por um dos casos relatados em que o uso do suplemento só foi informado após o diagnóstico clínico-laboratorial de intoxicação por vitamina D.

Em um dos casos, a intoxicação foi consequência do uso intramuscular de um composto vitamínico lipofílico de finalidade diversa àquela utilizada. Este caso representa um risco grave de uma prática que vem se disseminando em praticantes de musculação com o uso inadequado de uma droga de preparação veterinária (16). Afora o inerente 
risco de automedicação, a liberação da aplicação IM é lenta e, portanto, prolonga a intoxicação por vitamina $\mathrm{D}$.

$\mathrm{O}$ uso destas injeçóes intramusculares com finalidade estética de aumento do volume muscular no local da aplicação, sem correspondente hipertrofia, parece vinculado ao veículo oleoso utilizado e a intoxicação seria um "efeito colateral indesejado". Mas é importante observar que a hipervitaminose não é o único risco deste tipo de aplicação, podendo ocorrer eventos embólicos, perda da mobilidade do músculo, além de uma série de eventos locais como necrose e ulceraçóes. Existem poucos relatos desta prática na literatura médica, mas ela provavelmente é mais frequente do que se imagina (16).

Abordar a automedicação não é simples por uma série de motivos. Em primeiro lugar, não temos uma dimensão adequada do problema e mensurar a frequência disso pode ser extremamente complexo pela vergonha que alguns têm de admitir a prática. Por outro lado, profissionais de saúde devem participar ativamente da conscientização da populaçáo sobre os riscos da automedicaçáo (15), pois não existem panaceias (8) e mesmo inocentes suplementos vitamínicos podem ter consequências graves como aqui ilustrado.

\section{Conclusóes}

Os casos aqui relatados são exemplos do risco associado ao uso indiscriminado de vitamina $\mathrm{D}$, como prescrição ou suplemento, com apresentaçóes clínicas ameaçadoras à vida. É fundamental que a comunidade médica e científica participe ativamente da educação e orientação da população sobre os riscos da automedicação, mesmo que o uso de uma vitamina pareça algo inócuo.

\section{REFERÊNCIAS}

1. Ross et al. Dietary Reference Intakes for Calcium and Vitamin D. National Academies Press (US); 2011.

2. Herrmann et al. Assessment of vitamin D status - a changing landscape. Clin Chem Lab Med. 2017; 55(1): 3-26.

3. Marcinowska-Suchowierska et al. Vitamin D Toxicity - A Clinical Perspective. Frontiers in Endocrinology. 2018. 9: 550.

4. Jones G. Pharmacokinetics of vitamin D toxicity. Am J Clin Nutr. 2008;88(2):582S-586S.

5. Nesterova et al. 1,25-(OH)2D-24 Hydroxylase (CYP24A1) Deficiency as a Cause of Nephrolithiasis. Clin J Am Soc Nephrol. 2013; 8(4): 649-57.

6. Auguste BL, Avila-Casado C, Bargman JM. Use of vitamin $\mathrm{D}$ drops leading to kidney failure in a 54-year-old man. CMAJ. 2019;191(14):E390-E394.

7. Moreira et al. Reference values of 25-hydroxyvitamin D revisited. Arch 
Endocrinol Metab. 2020; 64 (4).

8. Benlidayi. Is vitamin $\mathrm{D}$ a panacea? Rheumatology International. 2020; 40: 821-822.

9. Galior, Grebe \& Singh. Development of Vitamin D Toxicity from

Overcorrection of Vitamin D

Deficiency: A Review of Case Reports. Nutrients. 2018; 10 (8): 953.

10. Ghauri et al. Redundancy is of no good; iatrogenic hypervitaminosis $\mathrm{D}$ : a rare case of persistent vomiting due to hypercalcemia. Clinical Medicine Insights: Case Reports. 2019; 12: 1-3.

11. Rooney et al. Trends in Use of High-Dose Vitamin D Supplements Exceeding 1000 or 4000 International Units Daily, 1999-2014. JAMA. 2017; 317 (23).

12. Zhou et al. Extreme hypercalcaemia due to accidental vitamin $\mathrm{D}$ intoxication. Journal of Pediatrics and Child Health; 2019; 55: 104-106.

13. Sanders et al. Annual high-dose oral vitamin $\mathrm{D}$ and falls and fractures in older women: a randomized controlled trial. JAMA. 2010; 303 (18): 1815-22.

14. Wallace et al. Urinary tract stone occurrence in the Women's Health Initiative (WHI) randomized clinical trial of calcium and vitamin D supplements. The American Journal Clinical Nutrition. 2011; 94 (1): 270-7.
15. Montastruc J-L, et al.

Pharmacovigilance, risks and adverse effects of self-medication. Therapie (2016)

16. Figueiredo et al. Cosmetic Doping: the Problems of Intramuscular Application of Oils. Rev Bras Med Esporte. 2011; 17(1): 56-61.

\section{Endereço para correspondência \\ Renato Demarchi Foresto \\ Núcleo de Ensino e Pesquisa \\ Fundaçáo Oswaldo Ramos - Hospital do Rim Rua Borges Lagoa, 960 - Vila Clementino. CEP: 04038-002 - São Paulo - SP - Brasil}

\section{TABELAS}

Tabela 1. Resultados de exames laboratoriais do paciente do Caso 1 no diagnóstico da intoxicação, alta hospitalar e seguimento ambulatorial.

\begin{tabular}{|l|l|l|l|l|}
\hline Exames & $\begin{array}{l}\text { Valores de } \\
\text { Referência }\end{array}$ & Diagnóstico & $\begin{array}{l}\text { Alta } \\
\text { hospitalar }\end{array}$ & $\begin{array}{l}\text { Seguimento } \\
12 \text { meses }\end{array}$ \\
\hline $\begin{array}{l}\text { Leucócitos } \\
\text { (N/mm3) }\end{array}$ & $\begin{array}{l}3.500- \\
10.500\end{array}$ & 8.900 & 6.000 & 7.000 \\
\hline $\mathrm{Hb}(\mathrm{g} / \mathrm{dL})$ & $13,5-17,5$ & 10,6 & 10,4 & 10,6 \\
\hline $\mathrm{Ht}(\%)$ & $36,0-50$ & 30,9 & 29,7 & 30,2 \\
\hline $\mathrm{PLT}(\mathrm{N} / \mathrm{mm} 3)$ & $\begin{array}{l}150.000- \\
450.000\end{array}$ & 266.000 & 245.000 & 243.000 \\
\hline $\begin{array}{l}\mathrm{Creat}(\mathrm{mg} / \\
\mathrm{dL})\end{array}$ & $0,80-1,20$ & 2,34 & 1,54 & 1,04 \\
\hline $\mathrm{Cai}(\mathrm{mmol} / \mathrm{L})$ & $1,11-1,40$ & 2,03 & 1,77 & - \\
\hline $\mathrm{CaT}(\mathrm{mg} / \mathrm{dL})$ & $8,8-10,6$ & 13 & - & 9,6 \\
\hline $\mathrm{P}(\mathrm{mg} / \mathrm{dL})$ & $2,4-4,6$ & 3,7 & - & 2,4 \\
\hline $\mathrm{K}(\mathrm{mEq} / \mathrm{L})$ & $3,6-5,0$ & 3,5 & 3,4 & 3,9 \\
\hline $\mathrm{PTH}(\mathrm{pg} / \mathrm{mL})$ & $15-68$ & 6 & 3 & 44 \\
\hline $\begin{array}{l}25 \mathrm{~mL}(\mathrm{pg} / \\
\mathrm{mL})\end{array}$ & $30-60$ & $>160$ & 145 & 61 \\
\hline
\end{tabular}


Hb: Hemoglobina; Ht: hematócrito; PLT: plaquetas; Creat: creatinina; Cai: cálcio iônico; CaT: cálcio total; P: fósforo; $K$ : potássio; PTH: paratormônio; 25OHD: 25-hidroxivitamina D.

Tabela 2. Resultados de exames laboratoriais do caso 2 no diagnóstico de intoxicação e no momento da alta hospitalar.

\begin{tabular}{|l|l|l|l|}
\hline Exames & $\begin{array}{l}\text { Valores de } \\
\text { Referência }\end{array}$ & Diagnóstico & Alta hospitalar \\
\hline $\begin{array}{l}\text { Leucócitos (N/ } \\
\mathrm{mm} 3)\end{array}$ & $3.500-10.500$ & 9.300 & 10.600 \\
\hline $\mathrm{Hb}(\mathrm{g} / \mathrm{dL})$ & $13,5-17,5$ & 12,5 & 10,9 \\
\hline $\mathrm{Ht}(\%)$ & $36,0-50$ & 35,1 & 31,2 \\
\hline $\mathrm{PLT}(\mathrm{N} / \mathrm{mm} 3)$ & $\begin{array}{l}150.000- \\
450.000\end{array}$ & 141.000 & 222.000 \\
\hline Creat $(\mathrm{mg} / \mathrm{dL})$ & $0,80-1,20$ & 4,20 & 3,34 \\
\hline Cai (mmol/L) & $1,11-1,40$ & 1,99 & 1,59 \\
\hline $\mathrm{CaT}(\mathrm{mg} / \mathrm{dL})$ & $8,8-10,6$ & 12,3 & - \\
\hline $\mathrm{P}(\mathrm{mg} / \mathrm{dL})$ & $2,4-4,6$ & 4,5 & - \\
\hline $\mathrm{K}(\mathrm{mEq} / \mathrm{L})$ & $3,6-5,0$ & 4,6 & 3,9 \\
\hline $\mathrm{PTH}(\mathrm{pg} / \mathrm{mL})$ & $15-68$ & 9 & - \\
\hline $\begin{array}{l}25 \mathrm{OHD}(\mathrm{pg} / \\
\mathrm{mL})\end{array}$ & $30-60$ & $>160$ & - \\
\hline
\end{tabular}

Hb: Hemoglobina; Ht: hematócrito; PLT: plaquetas; Creat: creatinina; Cai: cálcio iônico; CaT: cálcio total; P: fósforo; $K:$ potássio;

PTH: paratormônio; 25OHD: 25-hidroxivitamina D.

Tabela 3. Exames laboratoriais do caso 3 no momento do diagnóstico da intoxicação por vitamina $\mathrm{D}$, alta hospitalar e seguimento ambulatorial.

\begin{tabular}{|l|l|l|l|l|}
\hline Exames & $\begin{array}{l}\text { Valores de } \\
\text { Referência }\end{array}$ & Diagnóstico & $\begin{array}{l}\text { Alta } \\
\text { hospitalar }\end{array}$ & $\begin{array}{l}\text { Seguimento } \\
\text { 04 meses }\end{array}$ \\
\hline $\begin{array}{l}\text { Leucócitos (N/ } \\
\mathrm{mm} 3)\end{array}$ & $\begin{array}{l}3.500- \\
10.500\end{array}$ & 9130 & & \\
\hline $\mathrm{Hb}(\mathrm{g} / \mathrm{dL})$ & $13,5-17,5$ & 12,1 & & \\
\hline $\mathrm{Ht}(\%)$ & $36,0-50$ & 36,3 & & \\
\hline $\mathrm{PLT}(\mathrm{N} / \mathrm{mm} 3)$ & $\begin{array}{l}150.000- \\
450.000\end{array}$ & & & \\
\hline Creat (mg/dL) & $0,80-1,20$ & 1,49 & 0,95 & 0,81 \\
\hline
\end{tabular}

\begin{tabular}{|l|l|l|l|l|}
\hline Cai (mmol/L) & $1,11-1,40$ & 2,03 & 1,63 & 1,29 \\
\hline CaT $(\mathrm{mg} / \mathrm{dL})$ & $8,8-10,6$ & & - & \\
\hline $\mathrm{P}(\mathrm{mg} / \mathrm{dL})$ & $2,4-4,6$ & & - & \\
\hline $\mathrm{K}(\mathrm{mEq} / \mathrm{L})$ & $3,6-5,0$ & & & \\
\hline $\mathrm{PTH}(\mathrm{pg} / \mathrm{mL})$ & $15-68$ & 16,1 & - & \\
\hline $25 \mathrm{OHD}(\mathrm{pg} / \mathrm{mL})$ & $30-60$ & $>160$ & - & $>160$ \\
\hline
\end{tabular}

Hb: Hemoglobina; Ht: hematócrito; PLT: plaquetas; Creat: creatinina; Cai: cálcio iônico; CaT: cálcio total; P: fósforo; $K$ : potássio; PTH: paratormônio; 25OHD: 25-hidroxivitamina D.

Tabela 4. Exames laboratoriais do caso 4 no diagnóstico da intoxicação, alta hospitalar e seguimento ambulatorial.

\begin{tabular}{|c|c|c|c|c|}
\hline Exames & $\begin{array}{l}\text { Valores de } \\
\text { Referência }\end{array}$ & Diagnóstico & $\begin{array}{l}\text { Alta } \\
\text { hospitalar }\end{array}$ & $\begin{array}{l}\text { Seguimento } \\
18 \text { meses }\end{array}$ \\
\hline $\begin{array}{l}\text { Leucócitos } \\
\text { (N/mm3) }\end{array}$ & $\begin{array}{l}3.500- \\
10.500\end{array}$ & & & \\
\hline $\mathrm{Hb}(\mathrm{g} / \mathrm{dL})$ & $13,5-17,5$ & & & \\
\hline $\mathrm{Ht}(\%)$ & $36,0-50$ & & & \\
\hline $\begin{array}{l}\text { PLT (N/ } \\
\text { mm3) }\end{array}$ & $\begin{array}{l}150.000- \\
450.000\end{array}$ & & & \\
\hline $\begin{array}{l}\text { Creat (mg/ } \\
\mathrm{dL} \text { ) }\end{array}$ & $0,80-1,20$ & 3,10 & 1,41 & 1,14 \\
\hline $\begin{array}{l}\text { Cai } \\
\text { (mmol/L) }\end{array}$ & $1,11-1,40$ & 2,03 & 1,60 & \\
\hline $\begin{array}{l}\mathrm{CaT}(\mathrm{mg} / \\
\mathrm{dL})\end{array}$ & $8,8-10,6$ & & 12,1 & \\
\hline$P(\mathrm{mg} / \mathrm{dL})$ & $2,4-4,6$ & & - & \\
\hline $\mathrm{K}(\mathrm{mEq} / \mathrm{L})$ & $3,6-5,0$ & & & \\
\hline $\begin{array}{l}\text { PTH (pg/ } \\
\mathrm{mL})\end{array}$ & $15-68$ & 9 & - & \\
\hline $\begin{array}{l}25 \mathrm{OHD} \\
(\mathrm{pg} / \mathrm{mL})\end{array}$ & $30-60$ & $>160$ & - & 30 \\
\hline
\end{tabular}

Hb: Hemoglobina; Ht: hematócrito; PLT: plaquetas; Creat: creatinina; Cai: cálcio iônico; CaT: cálcio total; P: fósforo; $K$ : potássio; PTH: paratormônio; 25OHD: 25-hidroxivitamina D. 